\title{
Danza folklórica ecuatoriana: estudio comparativo para mejorar su enseñanza
}

\author{
Ecuadorian folk dance: a comparative study to improve its teaching
}

\author{
Walter Aguilar-Chasipanta *; Ana Bayas-Cano *; Edith Valarezo-Mendoza * \\ Carlos Galarza-Guamanquispe **
}

* Universidad Central del Ecuador, Ecuador; Unidad Educativa Mario Cobo, Ecuador | geo aguilar@yahoo.com

\section{PALABRAS CLAVE}

Agilidad

Folklor

Gesto

Medidas corporales

Motriz-artístico

Movimientos

\section{KEYWORDS}

Agility

Folklore

Gesture

Body measurements

Motor-artistic

Movements

\section{RESUMEN}

El objetivo de la presente investigación es realizar un estudio comparativo del gesto motriz-artístico entre 10 bailarines experimentados y otros 10 novatos del Grupo Folclórico de Danza Tradicional “Tungurahua”. Se registraron diferentes medidas corporales: edad, talla, peso; también se tomó como referencia los años de permanencia en el grupo, años con el mismo coreógrafo y tiempo semanal de repasos. Se realizaron mediciones de: distancia propulsiva, distancia de vuelo, altura de impulso vertical, elevación de las piernas en plie, ángulo de cadera con relación al salto y ángulo de rodilla con relación al salto, en el paso básico de Sanjuanito "Chicha Agria”. El análisis puso de manifiesto que, mientras más años se encuentran dentro de la actividad van perfeccionado los gestos, pero van perdiendo agilidad en cuanto a ángulos y elevaciones, mostrando por otro lado que los novatos por el mismo hecho de tener menor edad y menos años en el grupo, tienen mayor agilidad pero en contraste manifiestan una técnica errónea con ángulos y elevaciones defectuosas en el gesto motriz-artístico. Con este estudio diferenciado se logró detectar deficiencias, lo que permitirá diseñar mecanismo que orienten a una mejor enseñanza de ejecución de movimientos motrices-artísticos, en los jóvenes que incursionan en esta actividad artística.

\section{ABSTRACT}

The objective of this investigation is to make a comparative study from the artistic driving gesture between 10 experimented dancers and 10 beginners from the "Tungurahua" traditional folkloric group dance. Different corporal measures were registered: age, size, weight. Moreover the continuity in the group, the number of years with the same choreographer and the amount of rehearsal per week were considered as a reference. A variety of measures were taken: distance propelling measurement, flying distance, vertical altitude force, the leg's elevation from the foot, hip angle related to the knee's angle and related to jumping, in Sanjuanito "Chicha Agria” basic step. The analysis showed that the more experienced the person, the better the gestures, but their agility as regards angles and elevations gets worse. On the other hand, it showed that the beginner dancers, due to their age, are more agile but they make mistakes in their techniques of angles and elevations in the artistic-driving gesture. This differentiated study helped us to find some deficiencies so as to design mechanisms that will guide a better teaching by executing driving artistic movements in young people that start in the artistic activity. 


\section{Introducción}

Ecuador, asiento inconfundible de pueblos diversos que han guardado su originalidad hasta la fecha, de donde se rescatan culturas y tradiciones (escritas, orales y expresivas) que se mantienen de generación en generación. En él, la danza es una de sus expresiones más sublime, en la que el lenguaje corporal manifiesta las vivencias, sentimientos, sensaciones, ideas y pensamientos que se expresan en formas de rituales, danzas y juegos. Es fundamental para el desarrollo de capacidades musicales, artísticas, creativas, emocionales y fundamentalmente capacidades físicas y técnicas que son medibles para conocer su evolución, tanto en el ámbito cualitativo como cuantitativo que es el objeto de estudio del presente trabajo (Aguilar, 2011).

El aspecto mágico-religioso que el ser humano ha reflejado en las manifestaciones artísticas desde tiempos remotos es evidente; las pinturas rupestres, las danzas rituales, y por supuesto la música ritual. Los efectos tanto físicos como psicológicos de la danza en la sociedad, le permiten ser útil para muchas funciones. Puede ser una forma de adorar a los dioses, en tiempos arcaicos; un medio de honrar a nuestros ancestros o un método para crear magia, según la cultura de cada individuo. Se menciona la danza en la Biblia, y hasta la edad media era una parte usual de los homenajes y de las celebraciones religiosas. Aunque la Iglesia cristiana denunció la danza como inmoral, el cristianismo no consiguió suprimir todos los ritos paganos (Arteaga, 1997).

Según Brozas et al. (2011) los bailes folclóricos, los bailes tradicionales de regiones específicas, más que inventadas, son danzas que han evolucionado. A menudo conservan rasgos que en algún momento tuvieron significados mágicos o rituales. La danza puede ser recreativa, ritual o artística y va más allá del propósito funcional de los movimientos utilizados en el trabajo y los deportes para expresar emociones, estados de ánimo o ideas.

La música y la danza eran y siguen siendo elementos fundamentales en la relación de los habitantes, en ella se reúnen un número considerable de participantes, ya sea de manera recreacional como profesional, dedicándole solamente los fines de semana en alguna reunión social o dedicándole largas horas a su repaso, en los cuales se busca su una alta precisión que será demostrada en sus diferentes participaciones (Cruz Valle, 2009).

La danza va más allá del propósito funcional de los movimientos. La danza es la transformación de funciones normales y expresiones comunes en movimientos fuera de lo habitual (Marcial, 2005).

El cuerpo puede realizar acciones como rotar, doblarse, estirarse, saltar y girar. Variando estas acciones físicas y utilizando una dinámica distinta, los seres humanos pueden crear un número ilimitado de movimientos corporales. Dentro del extenso campo de movimientos que el cuerpo puede realizar, cada cultura acentúa algunos caracteres dentro de sus estilos dancísticos (Fructuoso Alemán, 2012; Gavazzo, 2006; Marcial, 2005).

Apoyados en el Programa Kinovea, una gran herramienta que nos permite estudiar sus movimientos (ángulos, distancias, alturas, etc.), para de esta manera notar las diferencias de ejecución tanto en profesionales como principiantes, entre hombres y mujeres, entre niños y niñas, proponemos un verdadero estudio comparativo para analizar sus diferentes movimientos y conseguir su posterior enseñanza con un perfeccionamiento que lo hará más vistosa en las diferentes presentaciones.

Es así que se estudió a los bailarines profesionales y novatos del Ballet Folklórico “Tungurahua”, un grupo muy representativo del Ecuador, analizando ángulos y elevaciones, logrando un acercamiento a un método para una correcta enseñanza.

Algunos estudios han demostrado que el aumento de la fuerza ocurre preferentemente en los grupos musculares que se entrenan con el ballet. De igual manera el equilibrio y la coordinación excelentes son muy 
importantes para los bailarines (son flexibles pero no hipomóviles). Se trata de una cualidad que a menudo se tiene en cuenta. Los bailarines con hiperflexibilidad son más propensos a sufrir lesiones, por ejemplo esquinces de tobillo, por ello hay quien considera esta condición como una desventaja (Castañer et al, 2008; Moya, 1995; Fructuoso Alemán, 2012).

Según Raimondi (1999) las diferencias más marcadas en la flexibilidad se encuentran en la cadera, tobillo y columna. Los bailarines han demostrado mayor movilidad de la columna, al igual que un mayor enderezamiento, disminuyendo de esta manera tanto la cifosis dorsal como la lordosis lumbar.

Desde la experiencia como profesionales de la danza, existe especial interés en este estudio, porque cada vez más los estudiantes y profesionales requieren un tratamiento más especializado. Cabe mencionar además que no se conoce de algún otro estudio al respecto, atreviéndonos a decir que el presente proyecto es el pionero en este tipo de investigaciones en este campo.

Por tanto, el objetivo de la presente investigación fue realizar un estudio comparativo del gesto motrizartístico entre 10 bailarines experimentados y otros 10 novatos del Grupo Folclórico de Danza Tradicional "Tungurahua”.

Este trabajo puede dar las pautas y nociones del arte folclórico para que se intuya, se aprenda con técnicas apropiadas y pedagógicas, para facilitar el aprendizaje porqué demanda mucho movimiento, elasticidad y coordinación en los distintos bailes acompañado con música y danza propia de los indígenas, mestizos y montubios del Ecuador, puesto que es un arte y su finalidad es transmitir al espectador sentimientos y sensaciones a través de los movimientos, pero no por ello ha de infravalorarse el esfuerzo físico que requiere. El entrenamiento y las demandas de la profesión son muy intensos, al igual que en el deporte profesional. El entrenamiento de la danza busca el desarrollo de amplitudes articulares máximas, junto con el incremento de la fuerza para poder mantenerlas.

\section{Metodologia}

\section{Participantes}

En el estudio participaron 20 bailarines profesionales de sexo masculino, con una edad promedio de 29,10 años (Desviación Típica -DT- $=11,78$ ), estatura promedio de 1,73 metros ( $\mathrm{DT}=0,07$ ), peso promedio de $63,50 \mathrm{Kg}$ (DT=7,68), años en el grupo promedio de 12,6 años (DT=7,67), años con el mismo coreógrafo de 12,6 años $(\mathrm{DT}=7,67)$, horas de repaso semanales promedio de 6 horas $(\mathrm{DT}=0)$, todos procedentes del grupo profesional; así como 10 bailarines novatos de sexo masculino, con edad promedio de 14,70 años (DT=1,68), estatura promedio de 1,47 metros ( $\mathrm{DT}=0,15)$, peso promedio de $50,40 \mathrm{Kg}(\mathrm{DT}=2,58)$, años en el grupo promedio de 0,25 años ( $\mathrm{DT}=0$ ), años con el mismo coreógrafo promedio de 0,25 años $(\mathrm{DT}=0)$, horas de repaso semanal promedio de 6 horas $(\mathrm{DT}=0)$, procedentes de las escuelas formativas del Ballet.

\section{Procedimiento}

Una vez efectuado el contacto con el Director del Ballet, se procedió a explicarle el objetivo de la investigación y conseguir su autorización respectiva para realizar las filmaciones a los bailarines profesionales como a los novatos de la escuela formativa. Una vez conseguida su autorización, se solicitó la colaboración de los sujetos a investigarse, explicándoles la naturaleza del estudio e indicándoles que se mantendrá el anonimato.

Para la presente investigación se usó la filmación, con los siguientes pasos: 
- Observación y análisis de los bailarines profesionales y novatos del Grupo “Tungurahua”.

- Ubicación de la cámara y el trípode a la altura indicada $(1,20 \mathrm{~m})$ y de manera perpendicular a los participantes.

- Distancia de filmación (3 m).

- Uso de ajuste automático para colores naturales.

- Exposición basada en todo el cuadro.

- Escena toma película.

- Resolución de pixeles: 1280x720 (fina).

- Proceso de filmación de cada bailarín. (Se realizó tres filmaciones por bailarín).

- Análisis y comparación de las filmaciones de los bailarines profesionales frente a los novatos usando el Programa Kinovea.

- Elaboración de las Tablas de Resultados.

\section{Instrumentos}

- Cámara Sony, Optical SteadyShot Optical Zoom 4x, 26 mm Wide-angle lens.

- Software utilizado: Youtube.com, Kinovea, Excel

- Hardware utilizado: Notebook, flash memory

- Trípode

- Cinta Métrica

- Masking

\section{Variables}

Las variables analizadas se las observa en la Figura 1, y fueron:

- Distancia propulsiva

- Distancia de vuelo

- Altura impulso vertical

- Elevación de la pierna en plie

- Ángulos de elevación de rodilla

- Ángulo de elevación de cadera

Figura 1: Gráfico de variables a ser medidas.

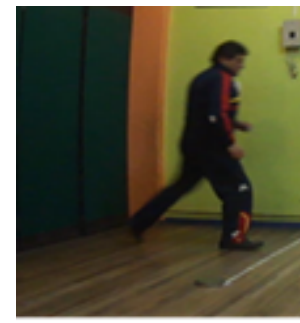

Distancia propulsiva

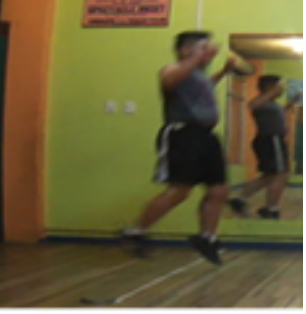

Distancia de vuelo

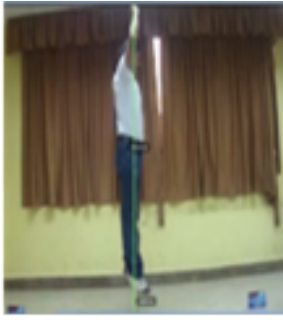

Altura en relación al piso

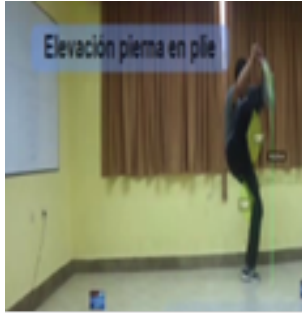

Plie

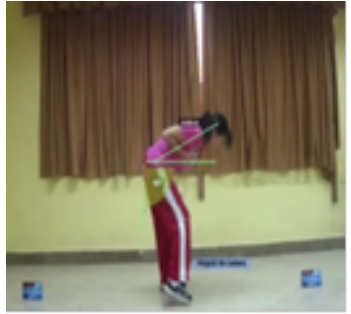

Ángulos de cadera y rodilla 


\section{Medidas}

Todos los bailarines fueron consultados en cuanto a sus datos de edad, talla, peso, tiempo de permanencia en el grupo, tiempo de permanencia con el mismo coreógrafo, horas de repaso a la semana. El resto de medidas fueron obtenidas mediante el Programa Kinovea.

\section{Sesiones de filmación}

Se las realizó en la Academia del Grupo Folklórico "Tungurahua”, ubicada en el Centro Comercial “Ambato”, lugar habitual de repasos del Ballet. Las filmaciones se realizaron en el Salón Principal de la Sede, los participantes debían ejecutar sus bailes un tiempo de 1 minuto cada uno, por tres ocasiones.

\section{Estadística}

Se empleó para esta investigación la Estadística Descriptiva usando las medidas de Tendencia Central y Dispersión, obteniendo resultados de la Mediana y la Desviación Típica respectivamente, de cada una de las variables analizadas, para lo cual se usó el Programa Excel 2010 del Paquete Microsoft Office Professional Plus 2010.

\section{Resultados}

En la Tabla 1, se muestra las características generales de los sujetos participantes en el estudio, distribuidos en profesionales y novatos, observando las siguientes diferencias significativas.

Tabla 1. Características generales por grupos

\begin{tabular}{|c|c|c|c|c|c|c|}
\hline \multicolumn{7}{|c|}{ CARACTERISTICAS GENERALES } \\
\hline & $\begin{array}{c}\text { EDAD } \\
\text { TIPNO DE BAILARÍN }\end{array}$ & $\begin{array}{c}\text { TALLA } \\
\text { (METRO } \\
\text { S) }\end{array}$ & PESO $(\mathrm{kg})$ & $\begin{array}{c}\text { AÑOS EN } \\
\text { EL } \\
\text { GRUPO }\end{array}$ & $\begin{array}{c}\text { AÑOS CON EL } \\
\text { MISMO } \\
\text { COREÓGRAFO }\end{array}$ & $\begin{array}{c}\text { TIEMPO DE } \\
\text { REPASO } \\
\text { SEMANAL } \\
\text { (Horas) }\end{array}$ \\
\hline $\begin{array}{c}\text { PROFESIONALES } \\
(\mathrm{n}=10)\end{array}$ & $29,10 \pm 11,78$ & $1,73 \pm 0,07$ & $63,50 \pm 7,68$ & $12,6 \pm 7,67$ & $12,6 \pm 7,67$ & $6 \pm 0,00$ \\
\hline NOVATOS (n=10) & $14,70 \pm 1,68$ & $1,47 \pm 0,15$ & $50,50 \pm 2,58$ & $0,25 \pm 0,00$ & $0,25 \pm 0,00$ & $6 \pm 0,00$ \\
\hline
\end{tabular}

Fuente: Elaborado por el autor

En la Figura No. 2, se observa el análisis por grupo investigado en cuanto a las características generales, poniendo de manifiesto diferencias significativas. 
Figura 2. Diferencia de datos generales de los grupos investigados

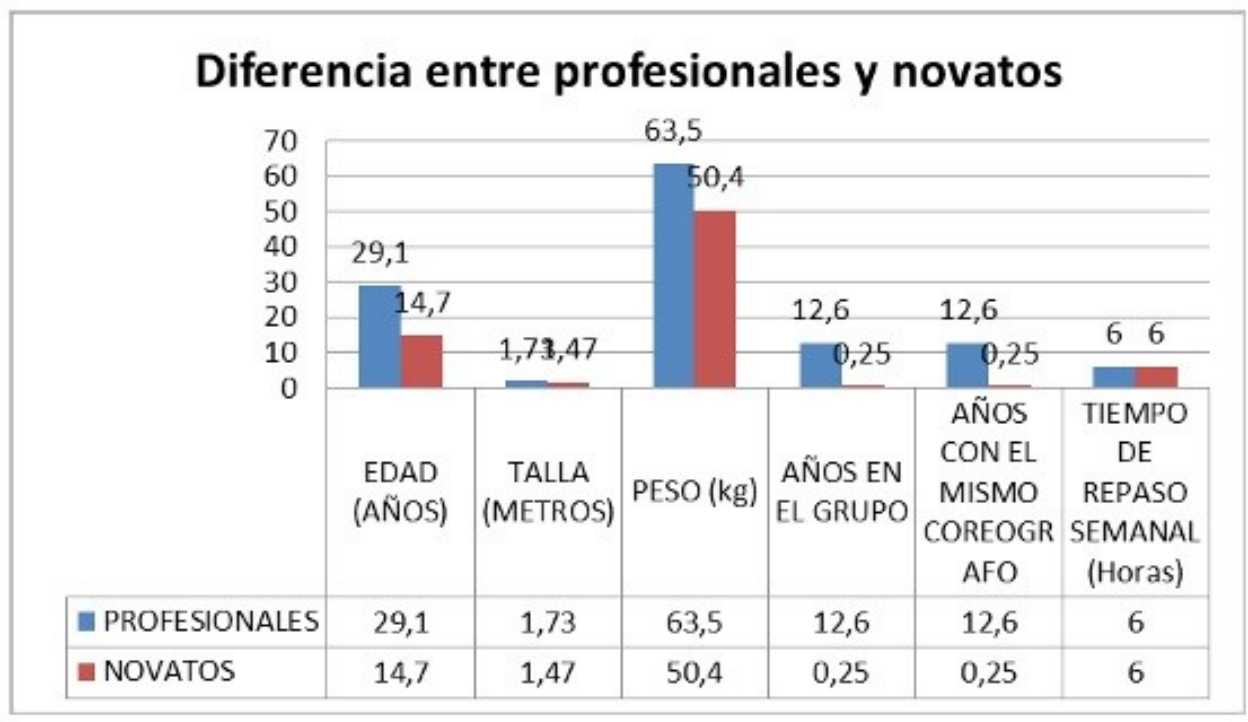

En la Tabla No. 2, se muestran los datos de la observación efectuada de las variables a ser medidas a los bailarines expertos y novatos de una manera cualitativa.

Tabla 2. Datos cualitativos de las variables

\begin{tabular}{|c|c|c|c|c|c|c|}
\hline \multicolumn{7}{|c|}{ DATOS DE LA OBSERVACIÓN DE LAS VARIABLES } \\
\hline TIPO DE BAILARÍN & $\begin{array}{c}\text { Distancia } \\
\text { Propulsiva }\end{array}$ & $\begin{array}{c}\text { Distancia } \\
\text { de vuelo }\end{array}$ & $\begin{array}{c}\text { Altura } \\
\text { impulso } \\
\text { vertical }\end{array}$ & $\begin{array}{c}\text { Elevación } \\
\text { pierna en } \\
\text { plie }\end{array}$ & $\begin{array}{c}\text { Ángulo } \\
\text { de cadera }\end{array}$ & $\begin{array}{c}\text { Ángulo de } \\
\text { rodilla }\end{array}$ \\
\hline PROFESIONALES & $\mathrm{xxx}$ & $\mathrm{xxx}$ & $\mathrm{xxx}$ & $\mathrm{xxx}$ & $\mathrm{xxx}$ & $\mathrm{xx}$ \\
\hline NOVATOS & $\mathrm{xx}$ & $\mathrm{xxx}$ & $\mathrm{xxx}$ & $\mathrm{xx}$ & $\mathrm{xx}$ & $\mathrm{xxx}$ \\
\hline
\end{tabular}

Fuente: Elaborado por el autor

En la Tabla 3, observamos los datos de las mediciones obtenidas en las variables motivo de análisis.

Tabla 3. Resultados medición de variables

\begin{tabular}{|c|c|c|c|c|c|c|}
\hline \multicolumn{7}{|c|}{ PROMEDIOS \pm DESVIACION ESTANDAR VARIABLES } \\
\hline TIPO DE BAILARÍN & $\begin{array}{c}\text { Distancia } \\
\text { Propulsiva } \\
\text { (cm) }\end{array}$ & $\begin{array}{c}\text { Distancia de } \\
\text { vuelo }(\mathrm{cm})\end{array}$ & $\begin{array}{c}\text { Altura } \\
\text { impulso } \\
\text { vertical } \\
\text { (cm) }\end{array}$ & $\begin{array}{l}\text { Elevación } \\
\text { pierna en } \\
\text { plie (cm) }\end{array}$ & $\begin{array}{l}\text { Ángulo de } \\
\text { cadera } \\
\text { (grados) }\end{array}$ & $\begin{array}{l}\text { Ángulo de } \\
\text { rodilla } \\
\text { (grados) }\end{array}$ \\
\hline PROFESIONALES & $21,43 \pm 10,38$ & $29,40 \pm 13,20$ & $6,40 \pm 1,96$ & $51,20 \pm 25,75$ & | $77,00 \pm 40,68$ & $118,00 \pm 56,56$ \\
\hline NOVATOS & $25,00 \pm 5,51$ & $31,49 \pm 7,65$ & $8,49 \pm 4,58$ & $56,61 \pm 9,55$ & $91,00 \pm 14,60$ & $109,00 \pm 15,85$ \\
\hline
\end{tabular}

Fuente: Elaborado por el autor 
En la Figura No. 3, se observa la diferencia de las medidas entre los dos grupos. Los valores más elevados corresponden a los profesionales, pero no es muy marcada la diferencia con los novatos.

Figura 3. Diferencia de datos de los grupos investigados

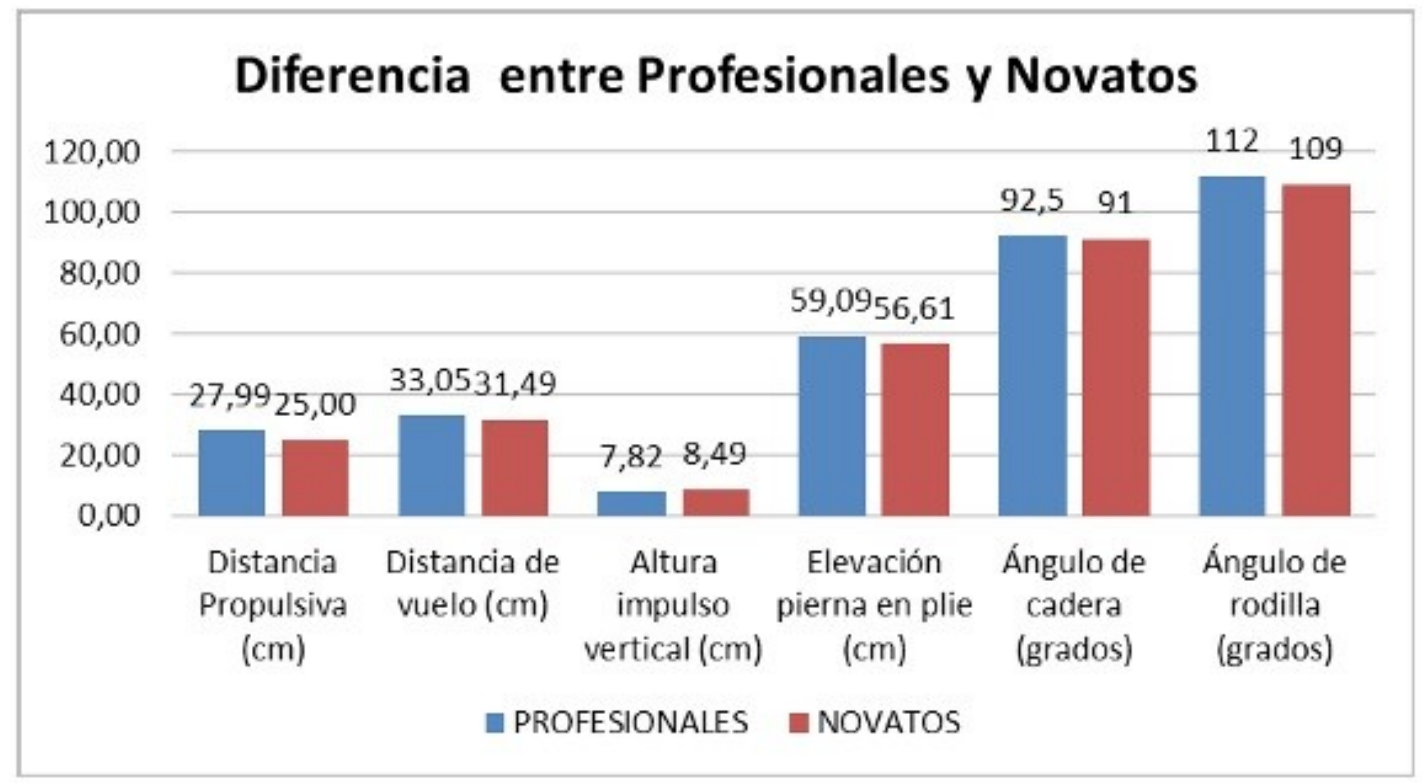

\section{Discusión}

La metodología utilizada, aun cuando ha sido sencilla, ha demostrado ser sensible y reproducible. Los resultados de este estudio muestran tal y como se esperaba, luego de analizados los datos proporcionados por los sujetos investigados así como los obtenidos mediante el uso del Programa Kinovea, un predominio no muy marcado de los bailarines profesionales, notando que, mientras más años permanezcan en el grupo, adquieren mayor perfección en la ejecución de la técnica correcta de los pasos de Sanjuanito en contraposición a los ángulos y distancias que muestran un retroceso; caso opuesto al de los novatos, quienes no muestran una técnica correcta, pero sí casi una homogeneidad en cuanto a los ángulos y distancias.

Algunas investigaciones han demostrado que al pasar los años, se va perdiendo la agilidad para realizar tal o cual movimiento, y dentro de la danza folklórica se requiere mucho de ella para poder efectuar varios pasos complicados. Por el contrario, los jóvenes llegan a su máximo nivel de agilidad en la pubertad (edad en la que se encuentran la mayoría de bailarines novatos investigados) y la mantienen varios años.

Todos los promedios de las variables (excepto el ángulo de rodilla) son más efectivas en los novatos.

\section{Conclusiones}

La experiencia juega un papel importante para la técnica de ejecución del gesto de la danza. La juventud juega un papel importante para la agilidad de movimientos. La sinergia de la experiencia y la juventud, dan como resultado un muy buen nivel de ejecución de los gestos, pues los novatos toman la experiencia de los profesionales y los mejoran, siendo un muy buen indicador para poder preparar una guía de enseñanza para niños y jóvenes que incursionan en este bello arte. 


\section{Agradecimientos}

Expresamos un profundo agradecimiento al Dr. Carlos Quinde Mancero, Director General del Grupo de Danza "Tungurahua”, por todas las facilidades prestadas para la ejecución de la presente investigación.

A los bailarines profesionales y novatos que colaboraron en la investigación, pues sin ellos no hubiese sido posible efectuar este trabajo.

\section{Referencias}

Aguilar, Walter, (2011). La Práctica de la Danza Folclórica en el desarrollo de valores culturales de los estudiantes del departamento de Cultura Física de la Universidad Técnica de Ambato en el período septiembre 2010 - febrero 2011”. (Tesis de Maestría). Universidad Técnica de Ambato, Ambato.

Arteaga, M. y Viciana, V. (1997) Desarrollo de la expresividad corporal, Barcelona: Inde.

Brozas Polo MP, García San Emeterio T, López Azcuna S. (2011). La danza contemporánea en España (1989-2009): aproximación a la creación coreográfica a través de la revista «Por la danza»Retos. Nuevas tendencias en Educación Física, Deporte y Recreación. 20, 16-20

Barboza Elizabeth. Manifestaciones folklóricas del estado Lara http://www.monografias.com/trabajos11/manfolk/manfolk.shtml

Carvalho, Neto Paulo de, (1961) “Folklor y Educación”, Editorial Casa de Cultura Ecuatoriana, Quito.

Cruz Valle, Nicolás. (2009) Danza folklórica, herramienta para la formación integral de los niños. Revista de Educación y Cultura. 47, 20

Fructuoso Alemán, Carolina (2012) La danza como elemento educativo en el adolescente. Apunts Educación Física y Deportes. 66, 31-37

García Ruso, H. (1997) La danza en la escuela, Barcelona: Inde.

Gavazzo Natalia. (2006). Las danzas de oruro en buenos aires: Tradición e innovación en el campo cultural boliviano. Cuadernos de la Facultad de Humanidades y Ciencias Sociales - Universidad Nacional de Jujuy, 31

Marcial Mary, Quinde Carlos. (2005). Texto de Folklore Ecuatoriano, Ambato.

Castañer M; Torrents C; Dinusová M ; Anguera MT. (2008). Habilidades motrices en expresión corporal y danza. Detección de t-patterns. Motricidad. European Journal of Human Movement, 21

Moya Colombia. (1995). Hacia una danza educativa. Perfiles Educativos, 68

Raimondi, P. (1999). Cinesiología y psicomotricidad, Barcelona: Paidotribo. 